\title{
Low-/high-dose-rate brachytherapy boost in patients with intermediate-risk prostate cancer treated with radiotherapy: long-term results from a single institution team experience
}

\author{
Silvia Rodríguez Villalba, MD, PhD', Paula Monasor Denia, MsC', Maria Jose Pérez-Calatayud, PhD², \\ Jose Richart Sancho, MsC 1.3, Jose Pérez-Calatayud, PhDl.4, Antonio Fuster Escrivá, PhD 5 , Pedro Torrus Tendero, PhD 5 , \\ Manuel Santos Ortega, PhD, MD' \\ 'Radiotherapy Department, Hospital Clinica Benidorm, Benidorm, Alicante, Spain, ${ }^{2}$ Radiotherapy Department, Fundación IVO Valencia, \\ València, Spain, ${ }^{3}$ Radiotherapy Department, Hospital Iniversitario San Juan, San Juan de Alicante, Alicante, Spain, ${ }^{4}$ Radiotherapy Department, \\ La Fe University and Polytechnic Hospital, Valencia, Spain, ${ }^{5}$ Urology Department, Marina Baixa Hospital, Villajoyosa, Alicante, Spain
}

\begin{abstract}
Purpose: To compare brachytherapy (BT) boost of low-dose-rate (LDR) and high-dose-rate (HDR) techniques in patients diagnosed with intermediate-risk prostate cancer.

Material and methods: Between January 2005 and February 2018, 142 patients (50 LDR and 92 HDR) with intermediate-risk prostate cancer were treated with a BT boost, and retrospectively analyzed. Prescribed dose was 45 Gy with external beam radiotherapy (EBRT) plus 100-108 Gy with LDR-BT, and 60 Gy with EBRT plus one fraction of 10 Gy with HDR-BT. 99\% of patients received androgen deprivation therapy (ADT) for 6 months. Primary endpoint was to compare LDR and HDR boosts in terms of biochemical progression-free survival (bPFS). Secondary endpoint, after re-classifying patients into "favorable" and "unfavorable" sub-groups, was to analyze differences with a similar treatment intensity.

Results: Median overall follow-up for the total cohort was 66.5 months (range, 16-185 months). There were no significant differences in bPFS, overall survival, cause specific survival, local failure, lymph node failure, or distant failure when LDR or HDR was employed. bPFS at 90 months was $100 \%$ for favorable, and $89 \%$ and $85 \%$ for unfavorable patients at 60 months and 90 months, respectively (log-rank test, $p=0.017$ ). The crude incidence of genitourinary acute and chronic toxicity grade 3 was $0.7 \%$ and $4 \%$, respectively. Twelve patients $(8 \%)$ had chronic rectal hemorrhage grade 2 , in whom argon was applied (4 LDR and 8 HDR).

Conclusions: Combined treatment is an excellent therapeutic option in patients with intermediate-risk prostate carcinoma, with similar results in both LDR and HDR approaches and very low toxicities. Importantly, the current literature has indicated that unfavorable-risk patients belong to a different category, and should be treated as patients with high-risk factors. Therefore, the stratification and identification of both risk groups is extremely relevant.
\end{abstract}

J Contemp Brachytherapy 2021; 13, 2: 135-144 DOI: https://doi.org/10.5114/jcb.2021.105280

Key words: prostate carcinoma, intermediate-risk, LDR-BT, HDR-BT.

\section{Purpose}

Intermediate-risk (IR) group for prostate carcinoma $(\mathrm{PCa})$ is probably the group that includes the most heterogeneous patients in this wide pathology. Radical prostatectomy, external beam radiotherapy (EBRT), or brachytherapy (BT) exclusively or in combination with or without androgen deprivation therapy (ADT), are the treatments recommended for this group of patients [1].
Dose escalation in PCa has demonstrated higher levels of prostate specific antigen (PSA) control rates [2-6], in specific trials with IR patients $[7,8]$.

In this manuscript, we present mono-institutional retrospective results from completed database of $\mathrm{PCa}$ patients diagnosed with IR, and then treated, in all cases, by the same team of professionals, using a combined treatment of EBRT and BT [low-dose-rate (LDR) ${ }^{125}$ I seeds permanent implants or high-dose-rate (HDR)]. 
To the best of our knowledge, this is the first comparative study dealing exclusively with IR PCa comparing LDR-BT and HDR-BT as a boost in the same institution. The main characteristics of this retrospective cohort was the uniformity of therapeutic decisions, treatment performed, and follow-up. All treatments were carried out by the same radiation oncologist. Moreover, the team of physicists followed a strict protocol to assure uniformity, which guaranteed that EBRT and BT contouring and technical process maintain a high degree of homogeneity.

The primary endpoint of this study was to compare the boost outcomes of two BT techniques, LDR and HDR, in terms of biochemical progression-free survival (bPFS) and toxicity. We have re-classified patients into "favorable" and "unfavorable" IR sub-groups (FIR and UIR) in order to identify potential result differences [9]. The secondary endpoint was to analyze any differences in both prognosis sub-groups that received treatments of similar intensities.

\section{Material and methods \\ Patient characteristics}

From January 2005 to February 2018, 188 patients diagnosed with IR PCa were treated with EBRT and BT in our department. Forty-six patients had no record of a post-BT PSA from a follow-up (since they were referred from other centers), and were therefore excluded from this study analysis (34 treated with LDR and 12 treated with HDR), leaving 142 patients eligible for inclusion. From January 2005 to June 2012, BT boost in IR patients was applied with LDR-BT, changing to HDR-BT boost when this prostate BT technique was incorporated in the department in July 2012. The Radiotherapy Department at our hospital is a reference center for patients from other clinics. Therapeutic decision is undertaken by various committees dealing with urological tumors in referring centers. Since 2005, the accepted protocol is a combined treatment of EBRT $+\mathrm{BT}+6$ months of ADT in patients diagnosed with IR PCa, according to the guidelines [1]. The cases treated exclusively with EBRT are based on personal decisions of patients or BT procedure contraindications. Between January 2005 and February 2018, 142 patients with a proven diagnosis of histopathologic IR PCa (D'Amico classification) [10] were treated with a BT boost with a complete follow-up (June 2020). The outcomes were then retrospectively analyzed.

All patients were included in IR based on baseline PSA (10-20 ng/ml), Gleason $\leq 7$, or stage T2b-T2c [evaluated with transrectal ultrasound and/or pelvic magnetic resonance imaging (MRI) and computed tomography (CT) scan of the abdomen and pelvis]. Patients were re-classified into FIR and UIR sub-groups. UIR was defined as the presence of 2 or 3 IR risk factors and/or Gleason $7(4+3)$ (ISUP, grade 3 ), and/or $\geq 50 \%$ of positive core biopsies.

\section{EBRT characteristics}

All patients were treated by EBRT with intensity-modulated radiotherapy therapy (IMRT), using seven statics fields with sliding-window technique or volumetric mod- ulated arc therapy (VMAT), when the latter became available in our institution (December 2013). In both cases, the conformity level and organs at risk (OARs) sparing were equivalent, VMAT was adopted due to its higher treatment delivery efficiency. Linac and treatment planning system were performed using a Clinac $2100 \mathrm{CD}$ and Eclipse, respectively (Varian, Palo Alto, USA). Clinical target volume (CTV) was the prostate and seminal vesicles. An additional margin (10 $\mathrm{mm}$ anterior, posterior, and lateral, and $8 \mathrm{~mm}$ superior and inferior) was added for planning target volume (PTV). The prescribed dose was $45 \mathrm{~Gy}(1.8 \mathrm{~Gy} / \mathrm{frac}-$ tion) using boost with LDR-BT and 60 Gy (2 Gy/fraction) with HDR-BT. A dose of 45 Gy with EBRT was administered in case of a boost with LDR-BT, as recommended by the ABS [11]. When the HDR-BT boost program commenced in our institution, the literature was first reviewed, and it was decided to select 60 Gy in 30 fractions [12-14]. EBRT was delivered in all patients before the BT boost.

\section{Brachytherapy characteristics}

Brachytherapy treatment was performed in all the patients using spinal/epidural anesthesia and sedation with an intra-operative procedure by a transperineally approach.

From January 2005 to June 2012, BT boost in IR patients was performed employing LDR-BT (permanent ${ }^{125}$ I seeds). The planning and seed delivery were performed with a transrectal ultrasound-guided (TRUS) unit (BK Medical Falcon 2101 EXL) in real-time using Fully Integrated Real time Seed Treatment (FIRST) system (Nucletron, Elekta AB, Stockholm, Sweden). The FIRST system consists of a seed Selectron-associated hardware and two software environments with an endocavity rotational mover (ECRM) attached to the needle stepper to acquire and reconstruct $3 \mathrm{D}$ volumetric data by rotating a bimodal ultrasound. The seed Selectron incorporates a diode array to verify a build sequence and assay seed source strength. Finally, this system utilizes a remote afterloader to deliver LDR ${ }^{125} \mathrm{I}$ seeds into the patient. The system provides an intra-operative interactive planning, virtual needle guidance, robotic seed delivery (loosed seeds), and needle retraction system. SPOT PRO (Nucletron, Elekta, Stockholm, Sweden) system was used for treatment planning.

Prostate gland (declared as CTV), urethra, and rectum were contoured. Dose was prescribed for the prostate, with a $2 \mathrm{~mm}$ isometric margin (except posteriorly, where no margin was applied) following ESTRO/EAU/EORTC recommendations $[15,16]$. The aim was to achieve a $D_{90}$ equal or higher to the $100 \%$ of prescribed dose, with $\mathrm{V}_{150 \%}$ $\leq 50 \%$ and $\mathrm{D}_{2 \mathrm{cc}} \leq 100 \%$ for the rectum. The prescribed dose was $100 \mathrm{~Gy}$ in 13 patients (26\%) and $108 \mathrm{~Gy}$ in the remaining 37 cases $(74 \%)$. The dose limit given to the urethra was constrained to $135 \%$ of the prescribed dose, based on a previous radiobiological study using a linear-quadratic model [17]. A post-plan was constructed for all patients one month after the implant, fusing an axial CT scan study (CVT ASIR, General Electric, Wisconsin, USA) and an MRI T2 axial sequence (1.5T MRI, General Electric, Wisconsin, USA). 
In July 2013, we commenced to employ a boost with real-time HDR-BT ( $\left.{ }^{192} \mathrm{Ir}\right)$. Treatment plans were created and optimized using a treatment planning system Oncentra Prostate (Elekta, Stockholm, Sweden). HDR boost was performed under real-time TRUS guidance. Plastic needles were inserted transperineally into the prostate. TRUS-based planning objectives included the prostate plus a $2 \mathrm{~mm}$ of margin (except posteriorly), with a $\mathrm{D}_{90}$ $\geq$ prescription dose, i.e., $>100 \%$, following GEC/ESTRO recommendations [18], keeping the urethra dose below $120 \%$ of the prescribed dose. Dose prescription was $10 \mathrm{~Gy}$ in 1 fraction brachytherapy procedure, which was executed on a MicroSelectron v 2 remote afterloader treatment system (Elekta, Stockholm, Sweden).

LDR-BT: Median number of seeds 55 (26-100). Median air kerma strength per seed $0.432 \mathrm{U}(0.305-0.660 \mathrm{U})$, being $1 \mathrm{U}=1 \mu \mathrm{Gym}^{2} \mathrm{~h}^{-1}$. Median intra-operative planning $\mathrm{D}_{90}$ was $115.25 \mathrm{~Gy}$ (100.3-135 Gy). The ${ }^{125}$ I source model used was a SelectSeed from Elekta, and calculations were based on TG-43 using AAPM consensus datasets.

HDR-BT: Median prostate $\mathrm{D}_{90}$ was $10.75 \mathrm{~Gy}$ (10.0511.7 Gy). This value was slightly higher than the prescription dose, prioritizing a coverage greater than $90 \%$. The ${ }^{192}$ Ir source model was a MicroSelectron HDR v2 from Elekta, and calculations were based on TG-43 using AAPM-ESTRO consensus datasets.

The same radiation oncologist, who performed EBRT treatments, completed brachytherapy procedures, unifying the technical and dosimetric criteria.

\section{Follow-up}

After the treatment, $100 \%$ of the patients had a personal follow-up by the same radiation oncologist. According to our departmental protocol, this involved patients having a 4 monthly PSA for the first year, and then every 6 months until fifth year, and then annually. PSA elevations of nadir +0.4 during follow-up and a normalization without any treatment was consider a bouncing phenomenon. Biochemical failure (BF) was defined as change of nadir +2 (Phoenix definition). When BF was evidenced, chest and abdomen $\mathrm{CT}$, pelvic MRI, bone scan, or choline positron emission tomography (PET-CT) was performed.

\section{Toxicity}

For toxicity analyses, the common terminology criteria for adverse events (CTCAE) version 4.0 was employed [19], examining acute toxicity in the first 3 months after the BT treatment. Toxicity was reported in every patient, with the same frequency described in the follow-up chart, which was retrospectively recoded.

\section{Statistical methods}

IBM SPSS Statistics version 15.0 (IBM, USA) was used for statistical analysis. Descriptive statistics were applied to compare prognostic factors. Baseline characteristics were compared using $t$-tests for continuous variables, assuming normality of the samples, and chi-square tests for categorical variables. Categorical variables were listed as frequencies and percentages, and continuous vari- ables were presented as medians (interquartile range). Kaplan-Meier method with log-rank test was used to calculate the actuarial bPFS, overall survival (OS), cause-specific survival (CSS), local failure (LF), lymph node failure (LNF), distant failure (DF), and comparisons. bPFS rate was calculated for all living patients. Cox proportional hazards regression model was utilized for univariable (UVA) and multivariable analysis (MVA) to assess factors associated with biochemical relapse-free survival. For the bPFS, OS, CSS, LF, LNF, and DF endpoints, UVA included age, percent positive cores (PPC), Gleason score ( $\leq 6$ vs. 7$)$, FIR vs. UIR, and the type of BT boost technique (LDR or HDR). We analyzed risk factors for toxicity comparing both techniques of BT boost. Cox proportional hazards analysis was performed to calculate odds ratios (ORs) and confidence intervals (CIs), evaluating the influence of patient's tumor and treatment characteristics on the risk of toxicity. MVA was performed using a logistic regression model. A $p$ value of $\leq 0.05$ was considered statistically significant.

Overall follow-up was calculated as the length of time taken to finish the combined radiotherapy treatment to the last contact follow-up.

\section{Results}

The median age of total cohort at diagnosis was 70 years (range, $51-81$ years). Twelve patients $(9 \%)$ were staged as T1c, 16 (11\%) T2a, 17 (12\%) T2b, and 97 (68\%) T2c. The median Gleason score was 6 (range, 2-7). Gleason score was $\leq 6$ in 64 patients (45\%), Gleason score was 7 in 63 patients $(44 \%)$, and in 15 patients $(11 \%)$, it was defined as well- or moderate-differentiated carcinoma. Median PSA was $9.2 \mathrm{ng} / \mathrm{ml}$ (range, 3.37-20 ng/ml). A total of $141(99 \%)$ patients received ADT for a median of 6 months (range, 6-24 months). Fifty patients were treated with LDR-BT (35\%) and 92 with HDR-BT (65\%). Clinical basal characteristics of both BT boost groups are described in Table 1.

Forty-two patients (30\%) were re-classified in FIR and $100(70 \%)$ in UIR. Of the last group, 86 patients $(86 \%)$ were due to 2-3 risk factors, 25 patients $(25 \%)$ with biopsies of Gleason $7(4+3)$, and 41 of 85 patients were the result of $\geq 50 \%$ positives biopsy cores. Twenty-two patients of the FIR group were treated with LDR-BT (52\%) and 20 with HDR-BT (48\%). Twenty-eight patients of the UIR group were treated with LDR-BT $(28 \%)$ and 72 with HDR-BT $(72 \%)$. Treatment characteristics of both BT techniques have been previously described.

The median overall follow-up (June 2020) for the entire cohort was 66.5 months (range, 16-185 months). The median follow-up was 117.5 months (range, 34-185 months) and 54 months (range, 16-90 months) for the LDR-BT and HDR-BT boost, respectively.

The median PSA at the last follow-up was $0.01 \mathrm{ng} / \mathrm{ml}$ (range, $0-132 \mathrm{ng} / \mathrm{ml}$ ) and $0.06 \mathrm{ng} / \mathrm{ml}$ (range, 0-65 ng/ml) for the LDR-BT and HDR-BT patients, respectively. Four patients $(8 \%)$ of the LDR-BT boost and 13 patients $(14 \%)$ of the HDR-BT boost experience a bouncing in a median time of 21.5 months (range, 18-24 months) and 12 months (range, 6-28 months), respectively. The median value of PSA elevation was $1.23 \mathrm{ng} / \mathrm{ml}$ (range, $0.90-1.97 \mathrm{ng} / \mathrm{ml}$ ) 
Table 1. Clinical characteristics at diagnostic of both BT groups (LDR and HDR)

\begin{tabular}{|c|c|c|c|}
\hline & $\begin{array}{c}\text { LDR-BT boost } \\
n=50\end{array}$ & $\begin{array}{c}\text { HDR-BT boost } \\
n=92\end{array}$ & $P$-value \\
\hline Age (years), median (range) & $69.5(54-80)$ & $70(51-81)$ & 0.333 \\
\hline \multicolumn{4}{|l|}{ Stage } \\
\hline T1c & $3(6 \%)$ & $9(10 \%)$ & 0.083 \\
\hline $\mathrm{T} 2 \mathrm{a}$ & $8(16 \%)$ & $8(9 \%)$ & 1.000 \\
\hline $\mathrm{T} 2 \mathrm{~b}$ & $3(6 \%)$ & $14(15 \%)$ & 0.008 \\
\hline $\mathrm{T} 2 \mathrm{C}$ & $36(72 \%)$ & $61(66 \%)$ & 0.011 \\
\hline Median Gleason (range) & $6(2-7)$ & $7(6-7)$ & 0.000 \\
\hline \multicolumn{4}{|l|}{ Gleason } \\
\hline$\leq 6$ & $34(68 \%)$ & $30(32 \%)$ & 1.000 \\
\hline WD & $5(10 \%)$ & $8(9 \%)$ & 0.405 \\
\hline$\overline{M D}$ & $1(2 \%)$ & $1(1 \%)$ & 1.000 \\
\hline Gleason 7 & $10(20 \%)$ & $53(58 \%)$ & \\
\hline $7(3+4)$ & $5(10 \%)$ & $34(37 \%)$ & 0.000 \\
\hline $7(4+3)$ & $5(10 \%)$ & $19(21 \%)$ & 0.003 \\
\hline PSA (ng/ml), median (range) & $9.58(3.37-20)$ & $9.2(3.8-20)$ & 0.631 \\
\hline Favorable intermediate-risk & $22(44 \%)$ & $20(22 \%)$ & 0.758 \\
\hline Unfavorable intermediate-risk & $28(56 \%)$ & $72(78 \%)$ & 0.000 \\
\hline \multicolumn{4}{|l|}{ Previous TUR } \\
\hline Yes & 0 & $4(4 \%)$ & \\
\hline No & $50(100 \%)$ & $88(96 \%)$ & 0.001 \\
\hline \multicolumn{4}{|l|}{ Previous open abdominal adenomectomy } \\
\hline Yes & 0 & $2(2 \%)$ & 0.001 \\
\hline No & $50(100 \%)$ & $90(98 \%)$ & \\
\hline \multicolumn{4}{|l|}{ Diabetes } \\
\hline Yes & $4(8 \%)$ & $22(24 \%)$ & 0.000 \\
\hline No & $46(92 \%)$ & $70(76 \%)$ & 0.026 \\
\hline \multicolumn{4}{|l|}{ Hypertension } \\
\hline Yes & $20(40 \%)$ & $49(53 \%)$ & 0.000 \\
\hline No & $30(60 \%)$ & $43(47 \%)$ & 0.128 \\
\hline \multicolumn{4}{|l|}{ Anticoagulation therapy } \\
\hline Yes & $5(10 \%)$ & $26(29 \%)$ & 0.000 \\
\hline No & $45(90 \%)$ & $66(71 \%)$ & 0.046 \\
\hline Volume prostate (cc) (MRI), median (range) & $42.2(12.5-102.6)$ & $60.5(14.47-167.46)$ & 0.005 \\
\hline \multicolumn{4}{|l|}{ Diagnosis MRI } \\
\hline Yes & $43(86 \%)$ & $87(95 \%)$ & 0.000 \\
\hline No & $7(14 \%)$ & $5(5 \%)$ & 0.564 \\
\hline ADT & $50(100 \%)$ & 91 (99\%) & 0.136 \\
\hline Neoadjuvant & $13(26 \%)$ & 39 (43\%) & 0.000 \\
\hline
\end{tabular}

$B T$ - brachytherapy, LDR - low-dose-rate, HDR - high-dose-rate, WD - well-differentiated, MD - moderate-differentiated, TURP - transurethral resection of the prostate, MRI - magnetic resonance imaging, ADT - androgen deprivation therapy

and $1.03 \mathrm{ng} / \mathrm{ml}$ (range, 0.42-2.6 ng/ml) in LDR-BT and HDR-BT groups, respectively.

bPFS was $93 \%$ and $91 \%$ at 60 and 90 months, respectively, in the total series. At 60 and 90 months, bPFS was $94 \%$ and $92 \%$ for LDR-BT boost and $92 \%$ and $92 \%$ for HDR-BT boost, respectively (log-rank test, $p=0.615$ ). Figure 1 illustrates bPFS for LDR-BT and HDR-BT boost patients. The Kaplan-Meier OS for the total series was 93\% and $84 \%$ at 60 and 90 months, respectively. The CSS were $98 \%$ at 60 months and $98 \%$ at 90 months. Ten patients $(7 \%)$ experienced $\mathrm{BF}, 4$ of them were treated with LDR-BT and the remaining 6 with HDR-BT. There was only one local failure in a patient treated with LDR-BT boost at 92 months after the treatment. This patient was salvaged with a second BT treatment with HDR ( 3 fractions of $10 \mathrm{~Gy}$ and 2 years of ADT). The patient is alive, with a biochem- 


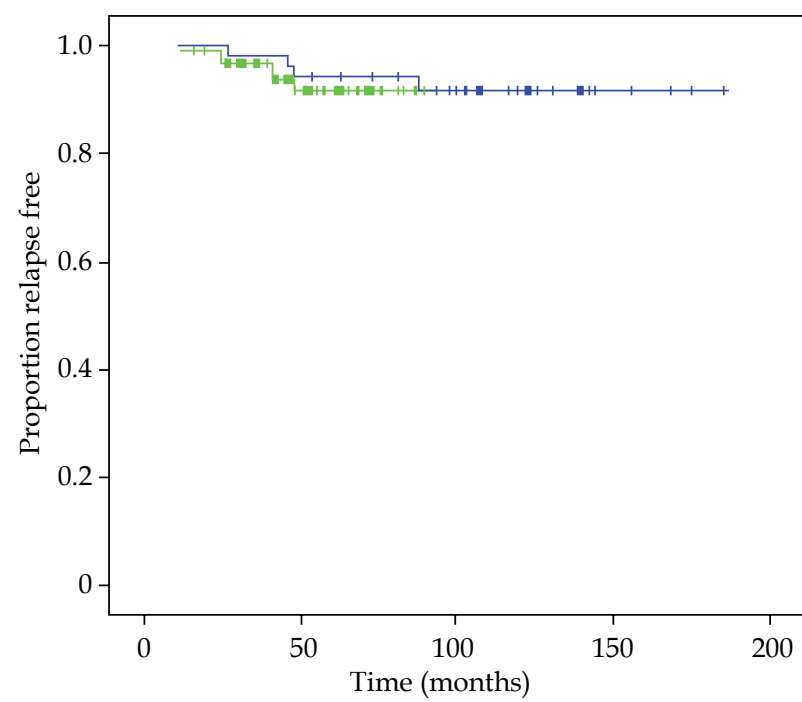

Fig. 1. Kaplan-Meier plot showing proportions of patients treated with LDR-BT boost (blue curve) and HDR-BT boost (green curve), remaining free of biochemical progression

ical control at 50 months after the salvage treatment. Five patients $(4 \%)$ developed lymph node failure, one treated with LDR-BT and the others 4 with HDR-BT. Five patients (3\%) developed metastatic disease, 2 patients in the LDR-BT group and 3 in the HDR-BT group. One patient treated with LDR-BT boost died with BF without an evidence of local or distant disease. In total, 20 non-prostate cancer deaths $(14 \%)$ were recorded during the follow-up period.

There was no significant difference in bPFS, OS, CSS, $\mathrm{LF}$, LNF, and DF between both techniques of BT boost. bPFS at 90 months was $100 \%$ for FIR patients and $89 \%$ and $85 \%$ for UIR at 60 months and 90 months, respectively (log-rank test, $p=0.017$ ). All patients with BF were included in the UIR group, $90 \%$ of them with 2 or 3 risk factors and $90 \%$ with PSA $\geq 10 \mathrm{ng} / \mathrm{ml}$. Figure 2 demonstrates bPFS for FIR and UIR patients. There was not significant difference in OS, CSS, local control, lymph node failure or distant failure between patients with FIR or UIR and in this last group between the techniques employed for the boost (Table 2).

Acute and chronic toxicity data are listed in Tables 3 and 4 . The highest acute and chronic genitourinary (GU) and gastrointestinal (GI) toxicities were recorded at each visit and graded from 0 to 5 according to CTCAE. The crude incidence of acute grade 3 GU toxicity was $0.7 \%$ (1 patient) in the LDR-BT boost group. There was no acute grade 3 GU toxicity when HDR-BT boost was employed. The crude incidence of chronic grade $3 \mathrm{GU}$ toxicity was $4 \%$ (2 LDR/4 HDR). One patient $(0.7 \%)$ treated with HDR-BT had an acute urinary retention needing a catheter for 10 days. Five patients (3.5\%) suffered from chronic urinary retention, which was solved with a transitory catheter; 3 of them were treated with LDR-BT boost and 2 with HDR-BT. Five patients (3.5\%), (2 LDR and 3 HDR) presented with urethral stricture grade 3; 1 patient was treated with transurethral resection of prostate (TURP)

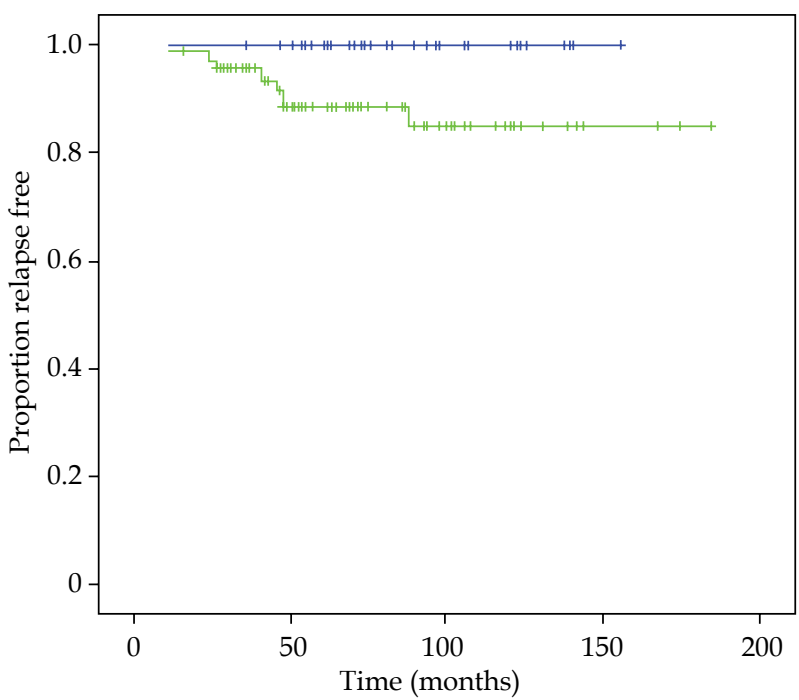

Fig. 2. Kaplan-Meier plot showing proportions of patients of favorable intermediate-risk (blue curve) and unfavorable intermediate-risk (green curve), remaining free of biochemical progression

and the other four with endoscopic urethrotomy. There was only one patient $(0.7 \%)$ treated with HDR who had chronic hematuria grade 3 . Chronic grade 1 rectal bleeding was superior $(p=0.03)$ in patients treated with HDR-BT. Twelve patients $(8 \%)$ needed cauterization with argon (grade 2) (4 LDR and 8 HDR) without statistical significance between the boost technique employed. There was no chronic grade 3 GI toxicity. No GU or GI acute or chronic grade 4-5 toxicity was observed.

\section{Discussion}

The 2017 American Society of Clinical Oncology (ASCO)/Cancer Care Ontario (CCO) brachytherapy guidelines state that brachytherapy boost (either LDR or HDR) should be offered to eligible IR PCa patients [20], based on three randomized trials [21-23]. These demon-

Table 2. Clinical results of favorable intermediaterisk (FIR) and unfavorable intermediate-risk (UIR) patients

\begin{tabular}{|c|c|c|c|}
\hline & $\begin{array}{c}\text { FIR } \\
n=42\end{array}$ & $\begin{array}{c}\text { UIR } \\
n=100\end{array}$ & $P$-value \\
\hline Biochemical failure & $0(0 \%)$ & $\begin{array}{c}10(10 \%) \\
\text { LDR boost } 4(4 \%) \\
\text { HDR boost } 6(6 \%)\end{array}$ & $\begin{array}{l}0.017 \\
0.892 \\
\end{array}$ \\
\hline Local failure & $0(0 \%)$ & $\begin{array}{c}1(1 \%) \\
\text { LDR boost } 1(1 \%) \\
\text { HDR boost } 0(0 \%)\end{array}$ & $\begin{array}{c}0.386 \\
-\end{array}$ \\
\hline Lymph node failure & $0(0 \%)$ & $\begin{array}{c}5(5 \%) \\
\text { LDR boost } 1(1 \%) \\
\text { HDR boost } 4(4 \%)\end{array}$ & 0.450 \\
\hline Distant failure & $0(0 \%)$ & $\begin{array}{c}4(4 \%) \\
\text { LDR boost } 2(2 \%) \\
\text { HDR boost } 2(2 \%)\end{array}$ & 0.511 \\
\hline
\end{tabular}

$L D R$ - low-dose-rate, $H D R$ - high-dose-rate 
Table 3. Acute toxicity (CTCAE v4) BT boost

\begin{tabular}{|c|c|c|c|c|c|c|}
\hline Toxicity & Grade & $\begin{array}{c}\text { LDR-BT boost } \\
n=50\end{array}$ & $\mathrm{D}_{90}$ mean $(\mathrm{Gy})$ & $\begin{array}{c}\text { HDR-BT boost } \\
n=92\end{array}$ & $\mathrm{D}_{90}$ mean (Gy) & $P$-value \\
\hline \multicolumn{7}{|l|}{ Urinary } \\
\hline \multirow[t]{4}{*}{ Dysuria } & 1 & $14(28 \%)$ & 113.26 & $25(27 \%)$ & 10.76 & 0.601 \\
\hline & 2 & $2(4 \%)$ & 120.86 & $14(15 \%)$ & 10.81 & 0.063 \\
\hline & 3 & $0(0 \%)$ & & $0(0 \%)$ & & - \\
\hline & $4-5$ & $0(0 \%)$ & & $0(0 \%)$ & & - \\
\hline \multirow[t]{4}{*}{ Frequency } & 1 & $6(12 \%)$ & 114.58 & $19(21 \%)$ & 10.08 & 0.292 \\
\hline & 2 & $23(46 \%)$ & 116.68 & $41(45 \%)$ & 10.52 & 0.399 \\
\hline & 3 & $0(0 \%)$ & & $0(0 \%)$ & & - \\
\hline & $4-5$ & $0(0 \%)$ & & $0(0 \%)$ & & - \\
\hline \multirow[t]{4}{*}{ Urgency } & 1 & $1(2 \%)$ & 113 & $14(15 \%)$ & 10.81 & 0.021 \\
\hline & 2 & $0(0 \%)$ & & $4(4 \%)$ & 10.67 & 0.157 \\
\hline & 3 & $0(0 \%)$ & & $0(0 \%)$ & & - \\
\hline & $4-5$ & $0(0 \%)$ & & $0(0 \%)$ & & - \\
\hline \multirow[t]{4}{*}{ Retention } & 1 & $13(26 \%)$ & 115.83 & $15(16 \%)$ & 10.03 & 0.075 \\
\hline & 2 & $2(4 \%)$ & 117.50 & $10(11 \%)$ & 10.82 & 0.211 \\
\hline & 3 & $0(0 \%)$ & & $0(0 \%)$ & & - \\
\hline & $4-5$ & $0(0 \%)$ & & $0(0 \%)$ & & - \\
\hline \multirow[t]{4}{*}{ Incontinency } & 1 & $0(0 \%)$ & & $3(3 \%)$ & 10.86 & 0.223 \\
\hline & 2 & $0(0 \%)$ & & $0(0 \%)$ & & - \\
\hline & 3 & $0(0 \%)$ & & $0(0 \%)$ & & - \\
\hline & $4-5$ & $0(0 \%)$ & & $0(0 \%)$ & & - \\
\hline \multirow[t]{4}{*}{ Hematuria } & 1 & $0(0 \%)$ & & $5(5 \%)$ & 10.70 & 0.112 \\
\hline & 2 & $0(0 \%)$ & & $0(0 \%)$ & & - \\
\hline & 3 & $1(2 \%)$ & 106 & $0(0 \%)$ & & 0.152 \\
\hline & $4-5$ & $0(0 \%)$ & & $0(0 \%)$ & & \\
\hline \multicolumn{7}{|l|}{ Gastrointestinal } \\
\hline \multirow[t]{4}{*}{ Diarrhea } & 1 & $1(2 \%)$ & 133.24 & $3(3 \%)$ & 10.99 & 0.721 \\
\hline & 2 & $0(0 \%)$ & & $0(0 \%)$ & & - \\
\hline & 3 & $0(0 \%)$ & & $0(0 \%)$ & & - \\
\hline & $4-5$ & $0(0 \%)$ & & $0(0 \%)$ & & - \\
\hline \multirow[t]{4}{*}{ Proctitis } & 1 & $7(14 \%)$ & 117.47 & 45 (49\%) & 10.73 & 0.000 \\
\hline & 2 & $2(4 \%)$ & 119.98 & $2(2 \%)$ & 10.53 & 0.477 \\
\hline & 3 & $0(0 \%)$ & & $0(0 \%)$ & & - \\
\hline & $4-5$ & $0(0 \%)$ & & $0(0 \%)$ & & - \\
\hline \multirow[t]{4}{*}{ Rectal bleeding } & 1 & $3(6 \%)$ & 119.32 & $5(5 \%)$ & 10.70 & 0.783 \\
\hline & 2 & $3(6 \%)$ & 105.77 & $1(1 \%)$ & 10.92 & 0.082 \\
\hline & 3 & $0(0 \%)$ & & $0(0 \%)$ & & - \\
\hline & $4-5$ & $0(0 \%)$ & & $0(0 \%)$ & & - \\
\hline
\end{tabular}

$B T$ - brachytherapy, $L D R$ - low-dose-rate, HDR - high-dose-rate

strate improved disease-free survival in patients treated with brachytherapy combined with external beam, compared to those treated with external beam exclusively.

Both techniques demonstrated their effectiveness, with certain advantages of HDR over LDR, including greater consistency of dose distribution, radiobiological effect (large dose per fraction), real-time planning, absence of seed migration, radiation protection improvement, and lower costs.

At present, there are only two published studies comparing LDR vs. HDR boost in prostate cancer, both including patients of intermediate- and high-risk [24, 25]. In King et al. [24] (18,403 patients of the National Cancer Database treated with BT boost), the results in terms of OS were similar without comparing toxicities, although they were based on retrospective inhomogeneous series, which the authors were aware of. For all patients analyzed, there were no differences between LDR and HDR boost, when sub-groups were divided by Gleason score, clinical T stage, NCCN risk category, or ADT. In a series of Slevin et al. [25] (287 patients of an institutional database), HDR boost was more than twice as likely to experience biochemical progression compared with LDR boost. At 5 years, bPFS was estimated to be $90.5 \%$ for the LDR-EBRT cohort, and $77.6 \%$ for the HDR-EBRT cohort. Cumulative incidence of $\geq$ grade $3 \mathrm{GU}$ and GI toxicities for the LDREBRT and HDR-EBRT cohorts were $8 \%$ vs. $4 \%$ and $5 \%$ vs. $1 \%$, respectively, although not statistically significant.

Even though, intermediate- and high-risk prostate tumors have different behaviors, both groups are treat- 
Table 4. Chronic toxicity (CTCAE v4) BT boost

\begin{tabular}{|c|c|c|c|c|c|c|}
\hline Toxicity & Grade & $\begin{array}{l}\text { LDR-BT boost } \\
n=50\end{array}$ & $\mathrm{D}_{90}$ mean (Gy) & $\begin{array}{l}\text { HDR-BT boost } \\
\quad n=92 \\
\end{array}$ & $\mathrm{D}_{90}$ mean (Gy) & $P$-value \\
\hline \multicolumn{7}{|l|}{ Urinary } \\
\hline \multirow[t]{4}{*}{ Dysuria } & 1 & $5(10 \%)$ & 111.47 & $2(2 \%)$ & 10.18 & 0.05 \\
\hline & 2 & $4(8 \%)$ & 114.72 & $3(3 \%)$ & 10.67 & 0.241 \\
\hline & 3 & $0(0 \%)$ & & $0(0 \%)$ & & - \\
\hline & $4-5$ & $0(0 \%)$ & & $0(0 \%)$ & & - \\
\hline \multirow[t]{4}{*}{ Frequency } & 1 & $3(6 \%)$ & 107.95 & $4(4 \%)$ & 10.53 & 0.649 \\
\hline & 2 & $9(18 \%)$ & 118.13 & $8(9 \%)$ & 10.57 & 0.212 \\
\hline & 3 & $0(0 \%)$ & & $0(0 \%)$ & & - \\
\hline & $4-5$ & $0(0 \%)$ & & $0(0 \%)$ & & - \\
\hline \multirow[t]{4}{*}{ Urgency } & 1 & $2(4 \%)$ & 107.50 & $6(7 \%)$ & 10.73 & 0.464 \\
\hline & 2 & $3(6 \%)$ & 124.74 & $7(8 \%)$ & 10.85 & 0.477 \\
\hline & 3 & $0(0 \%)$ & & $0(0 \%)$ & & - \\
\hline & $4-5$ & $0(0 \%)$ & & $0(0 \%)$ & & - \\
\hline \multirow[t]{4}{*}{ Retention } & 1 & $3(6 \%)$ & 108.83 & $5(5 \%)$ & 10.76 & 0.800 \\
\hline & 2 & $3(6 \%)$ & 118.38 & $2(2 \%)$ & 10.53 & 0.358 \\
\hline & 3 & $0(0 \%)$ & & $0(0 \%)$ & & - \\
\hline & $4-5$ & $0(0 \%)$ & & $0(0 \%)$ & & - \\
\hline \multirow[t]{4}{*}{ Incontinency } & 1 & $1(2 \%)$ & 105.00 & $2(2 \%)$ & 10.64 & 0.987 \\
\hline & 2 & $0(0 \%)$ & & $2(2 \%)$ & 10.75 & 0.397 \\
\hline & 3 & $0(0 \%)$ & & $0(0 \%)$ & & - \\
\hline & $4-5$ & $0(0 \%)$ & & $0(0 \%)$ & & - \\
\hline \multirow[t]{4}{*}{ Hematuria } & 1 & $4(8 \%)$ & 108.50 & $6(7 \%)$ & 10.62 & 0.996 \\
\hline & 2 & $2(4 \%)$ & 114.48 & $0(0 \%)$ & & 0.066 \\
\hline & 3 & $0(0 \%)$ & & $1(1 \%)$ & 10.90 & 0.157 \\
\hline & $4-5$ & $0(0 \%)$ & & $0(0 \%)$ & & - \\
\hline \multirow[t]{4}{*}{ Urethral stenosis } & 1 & $1(2 \%)$ & 117.00 & $2(2 \%)$ & 10.52 & 0.874 \\
\hline & 2 & $0(0 \%)$ & & $0(0 \%)$ & & - \\
\hline & 3 & $2(4 \%)$ & 112.48 & $3(3 \%)$ & 10.54 & 0.2771 \\
\hline & $4-5$ & $0(0 \%)$ & & $0(0 \%)$ & & - \\
\hline \multicolumn{7}{|l|}{ Gastrointestinal } \\
\hline \multirow[t]{4}{*}{ Diarrhea } & 1 & $1(2 \%)$ & 119.80 & $0(0 \%)$ & & 0.317 \\
\hline & 2 & $0(0 \%)$ & & $0(0 \%)$ & & - \\
\hline & 3 & $0(0 \%)$ & & $0(0 \%)$ & & - \\
\hline & $4-5$ & $0(0 \%)$ & & $0(0 \%)$ & & - \\
\hline \multirow[t]{4}{*}{ Proctitis } & 1 & $4(8 \%)$ & 118.17 & $4(4 \%)$ & 10.80 & 0.239 \\
\hline & 2 & $0(0 \%)$ & & $3(3 \%)$ & 10.73 & 0.228 \\
\hline & 3 & $0(0 \%)$ & & $0(0 \%)$ & & - \\
\hline & $4-5$ & $0(0 \%)$ & & $0(0 \%)$ & & - \\
\hline \multirow[t]{4}{*}{ Rectal bleeding } & 1 & $3(6 \%)$ & 122.66 & $18(20 \%)$ & 10.80 & 0.033 \\
\hline & 2 & $3(6 \%)$ & 115.24 & $9(10 \%)$ & 10.72 & 0.488 \\
\hline & 3 & $0(0 \%)$ & & $0(0 \%)$ & & - \\
\hline & $4-5$ & $0(0 \%)$ & & $0(0 \%)$ & & - \\
\hline
\end{tabular}

$B T$ - brachytherapy, LDR - low-dose-rate, HDR - high-dose-rate

ed equally with the same therapeutic intensity in most of the published studies, in which BT together with EBRT, constitute the therapeutic program. The use of different BT techniques, total dose, and dose per fraction, among others, are the data, which may influence clinical results and toxicity profiles. Analyzing published series with both risk groups, only three unique prospective randomized studies have been published comparing BT boost with EBRT $[21,23,26]$. All three were analyzed in a meta-analysis published in 2018, describing a significant benefit of 5-year biochemical control in favor of patients, for whom BT was used as a boost [27] being recommended for IR patients. For Sathya et al. [21], bPFS at 10 years was $67 \%$ in BT arm (LDR temporary ${ }^{192} \mathrm{Ir}$ ), for Hoskin et al. [23] analyses it was 46\% (HDR temporary ${ }^{192} \mathrm{Ir}$ ), and in ASCENDE-RT trial [26] (LDR permanent ${ }^{125}$ I) it was $83 \%$ at 9 years. These three different studies have different total EBRT doses and even fractionations, with the first two employing exclusive EBRT doses much lower than current standards. In addition to the above, randomized data from mature mono-institutional series with a long follow-up reports have been published with very favor- 
able results for patients treated with BT as a boost. All of them described high bPFS rates, between $84 \%$ and $91 \%$ in IR patients using either HDR [28-33] or LDR [34].

The published literature of combined treatment (EBRT plus LDR or HDR-BT) including IR patients exclusively is scarce [35-38]. In contrast to these data, our series reflects great homogeneity, both in the performance of the treatments and in the follow-up of these patients over time. Furthermore, all the treatments as well as clinical and technical data compilations were carried out by the same team. This provides uniformity in the criteria used in the handling of the data, eliminating "inter-observer" variables and delivering greater rigor to the observation. These data showed no differences when LDR-BT or HDR-BT was used, obtaining a bPFS of $92 \%$ at 90 months with both techniques, which are very similar to $91 \%$ at 5 years reached by Martell et al. [35] with HDR, and 87\% at 5 years by Chao et al. [36] with LDR.

There are difficulties and limitations in comparing our toxicity with published studies regarding the inhomogeneity of brachytherapy technique itself (real-time, CT planning, MRI fusion), different fractionations of EBRT, and differences in score systems (CTCAE, LENT-SOMA, RTOG scale). Even within the identical system, the events and terminology are different according to the version used (CTCAE v 4.0 or v3.0). The vast majority of our patients reported no side effects or grade 1-2, which corresponds to irritative voiding symptoms treatable by a-blockers or anticholinergic therapy.

Two, very relevant studies, have been published on the implication of BT in a combined treatment of PCa. The first of Hoskin et al. [23] with HDR, and the second, the ASCENDE-RT trial [39] using LDR as a boost. Both are cited as examples in the discussions of multiple publications with negative criticism regarding toxicity. In their study, Hoskin et al. employed an adapted version of a Dische scales for relating toxicity [23]. In the prospective study ASCENDE-RT, Rodda et al. [39] utilized a modified LENT-SOMA criteria, a system that includes multiple adverse effect factors in the same group without being broken down individually. Most of these factors would be classified at a lower degree if other toxicity scales were used. This is evident from the results not reproduced in prospective or retrospective studies, using the same doses in combined treatments.

We have searched publications, in which toxicity was defined based on CTCAE version 4.0 and patients were treated with similar schedules, finding equivalent results to our series. Sakurai et al. [40] analyzed 124 patients who underwent 13 Gy HDR-BT followed by EBRT (46 Gy/ 23 fractions), with the reported incidences of grade 2 urinary frequency and urinary retention in $45.5 \%$ and $18.1 \%$, respectively, and $3.3 \%$ of grade 2 rectal hemorrhage, as evaluated by lower gastrointestinal endoscopy observed in four patients. Büchser et al. [41] analyzed prospectively 210 patients treated with HDR-BT (15 Gy single fraction) plus three-dimensional conformal radiotherapy (3D-CRT) (37.5 Gy/15 fractions). The incidence of toxicity was very low. Grade 2 events were due to urethral strictures (3 patients), incontinence ( 3 patients), hematuria ( 2 patients), and retrograde ejaculation (1 patient). The incidence of late grade 2 and grade 3 GI toxicity was 5.2\% (11 patients) and $1 \%$ ( 2 patients), respectively. The authors concluded that the reason for these very low toxicities were partly explained by the use of real-time MRI-TRUS fusion for treatment planning. Kollmeier et al. [42] analyzed safety and toxicity utilizing LDR ${ }^{103} \mathrm{Pd}$ prostate brachytherapy in combination with ultra-hypofractionated radiotherapy (25 Gy delivered in 5 Gy fractions) in 46 patients with intermediate-risk prostate cancer. The most common grade 2 urinary toxicities investigated were urinary frequency/ urgency (25\%) and urinary obstruction/retention (10\%). No patients suffered from urinary retention requiring catheterization. Chao et al. [36] reported retrospectively 31 patients who received 45 Gy plus ${ }^{125}$ I boost with an incidence of late grade $3 \mathrm{GU}$ toxicity of $6.5 \%$ with urinary retention, with two patients requiring either a bladder neck incision (BNI) or TURP.

In the present study, despite the differences between the two groups (greater frequency of previous TURP and adenectomy, higher percentage of comorbidities, a threefold greater use of anticoagulants, larger prostate size in the HDR-BT boost patients and higher EBRT doses), no differences between them in terms of toxicity grade 2 or higher were noted. These aspects may explain the significant increase in grade $1 \mathrm{GU}$ toxicity and grade 1 rectal bleeding when HDR was used, not requiring treatment or being controlled with medication in all cases. We described $3.5 \%$ of urethral stenosis grade 3 that needed an elective operation, which was in the lower range of the published literature, as previously stated.

The only statistically significant factor in biochemical control was the differentiation between FIR and UIR. When we analyzed both groups separately, we found a 10 -year clinical and biochemical control in $100 \%$ of the patients with FIR, and patients with good prognosis factors, for which this data greatly improved the results obtained with exclusive treatments: EBRT, LDR, or HDR monotherapy [43-47].

Existing data differentiating patients into FIR and UIR are the key factor in prognosis, outcomes, and treatment selection. Patients with unfavorable intermediate-risk disease had a higher risk of PSA recurrence, local failure, development of metastases as well as death from prostate cancer, as described by Preisser et al. [48]. Due to this, a combined treatment of external beam and brachytherapy ( \pm androgen deprivation) is recommended [1].

In our series, bPFS in UIR was $85 \%$ at 90 months; biochemical failure was evident in 10 patients, 4 treated with LDR-BT and 6 with HDR, without statistically significant differences in terms of recurrence (lymph node or distant) and technique employed. Only one patient treated with seeds had a local failure. ADT addition, showed an increased bPFS in UIR patients $[49,50]$. In this homogeneous mono-institutional series, $99 \%$ of the patients received at least 6 months of ADT. None of the factors analyzed independently, including Gleason score $7(4+3),>50 \%$ of positives biopsies, perineural invasion, or 2-3 factors that define IR group, showed a statistical difference leading to greatest number of biochemical failures in this group. We hypothesize that the percentage of failures may be due to other technical aspects (total dose, no pelvic irradiation) 
or biological (genetic mutations) factors. A very important difference, when comparing our data with the outcomes of published series, is the wide difference of schemes in both treatment components (BT and EBRT), differences in doses and sometimes in volumes of treatments, such as 15 Gy combined with 37.5 Gy in 15 fractions [35, 51], 60 Gy with 9 Gy with HDR [45], 46 Gy plus 2 fractions of 11.5 Gy [33], 20/44 Gy combined with ${ }^{103} \mathrm{Pd}$ boost [52], or 46 Gy plus 115 Gy ${ }^{125} \mathrm{I}$ implant in the ASCENDE-RT trial [26]. Inevitably, this diversity represents a notable difference between the various "intensities" of respective programs, which, in our department, led us to consider that perhaps the dose selected in 2013 in combined treatments with HDR (60 Gy with EBRT and $10 \mathrm{~Gy}$ ) could be insufficient for patients with unfavorable intermediate-risk, and consequently, modifying the protocol based on the results of this analysis, adapting it to the more recent published recommendations [1].

Potential limitations of this study are the retrospective analyses and mono-institutional nature. It seems appropriate to emphasize the performance of more prospective studies in order to identify significant factors in the results, in an attempt to achieve greater control and more stable results in this particular group of patients.

\section{Conclusions}

In this mono-institutional and very homogeneous study, despite being retrospective, it is demonstrated that BT combined with EBRT is an excellent therapeutic option in patients with IR PCa, with equal results when both LDR and HDR techniques are employed, showing very low toxicities.

Patients included in UIR constitute a different entity, and should be treated as patients with high-risk factors. The stratification and identification of both risk groups is of great importance.

\section{Disclosure}

The authors report no conflict of interest.

\section{References}

1. Prostate cancer. NCCN Clinical Practice Guidelines in Oncology version 2.2020.

2. Pollack A, Smith LG, von Eschenbach AC. External beam radiotherapy dose response characteristics of 1127 men with prostate cancer treated in the PSA era. Int J Radiat Oncol Biol Phys 2000; 48: 507-512.

3. Beckendorf V, Guerif S, Le Prisé E et al. 70 Gy versus 80 Gy in localized prostate cancer: 5-year results of GETUG 06 randomized trial. Int J Radiat Oncol Biol Phys 2011; 80: 1056-1063.

4. Dearnaley DP, Jovic G, Syndikus I et al. Escalated-dose versus control-dose conformal radiotherapy for prostate cancer: Long-term results from the MRC RT01 randomised controlled trial. Lancet Oncol 2014; 15: 464-473.

5. Zelefsky M, Chana M, Yamada Y et al. Long-term outcome of high dose intensity modulated radiation therapy for patients with clinically localized prostate cancer. J Urol 2006; 176: 1415-1419.

6. Pollack A, Zagars GK, Starkschall G et al. Prostate cancer radiation dose response: results of the M. D. Anderson phase
III randomized trial. Int J Radiat Oncol Biol Phys 2002; 53: 1097-1105.

7. Heemsbergen WD, Al-Mamgani A, Slot A et al. Long-term results of the Dutch randomized prostate cancer trial: Impact of dose-escalation on local, biochemical, clinical failure, and survival. Radiother Oncol 2014; 110: 104-109.

8. Michalski JM, Moughan J, Purdy J et al. Effect of standard vs dose escalated radiation therapy for patients with intermediate-risk prostate cancer: The NRG Oncology RTOG 0126 Randomized Clinical Trial. JAMA Oncol 2018; 4: e180039.

9. Zumsteg ZS, Zelefsky MJ, Woo K et al. Unification of favourable intermediate-, unfavourable intermediate-, and very high-risk stratification criteria for prostate cancer. BJU Int 2017; 120: E87-E95.

10. D'Amico AV, Whittington R, Malkowicz SB et al. Biochemical outcome after radical prostatectomy, external beam radiation therapy, or interstitial radiation therapy for clinically localized prostate cancer. JAMA 1998; 280: 969-974.

11. Nag S, Beyer D, Friedland J et al. American Brachytherapy Society (ABS) recommendations for transperineal permanent brachytherapy of prostate cancer. Int J Radiat Oncol Biol Phys 1999; 44: 789-799.

12. Agoston P, Major T, Fröhlichet G et al. Moderate dose escalation with single-fraction high-dose-rate brachytherapy boost for clinically localized intermediate- and high-risk prostate cancer: 5-year outcome of the first 100 consecutively treated patients. Brachytherapy 2011; 10: 376-384.

13. Kovacs G, Potter R, Loch T et al. GEC/ESTRO-EAU recommendations on temporary brachytherapy using stepping sources for localised prostate cancer. Radiother Oncol 2005; 74: 137-148.

14. Pistis F, Guedea F, Pera J et al. External beam radiotherapy plus high-dose-rate brachytherapy for treatment of locally advanced prostate cancer: The initial experience of the Catalan Institute of Oncology. Brachytherapy 2010; 9: 15e22.

15. Ash D, Flynn A, Battermann J et al. ESTRO/EAU/EORTC recommendations on permanent seed implantation for localized prostate cancer. Radiother Oncol 2000; 57: 315-321.

16. Salembier C, Lavagnini $P$, Nickers $P$ et al. Tumour and target volumes in permanent prostate brachytherapy: a supplement to the ESTRO/EAU/EORTC recommendations on prostate brachytherapy. Radiother Oncol 2007; 83: 3-10.

17. Lliso F, Pérez-Calatayud J, Carmona V et al. Urethral dosimetry constraints in $125 \mathrm{I}$ permanent prostate brachytherapy used as boost to external radiotherapy. Rev Fis Med 2010; 11: 101-106.

18. Hoskin PJ, Colombo A, Henry A et al. GEC/ESTRO recommendations on high dose rate afterloading brachytherapy for localised prostate cancer: an update. Radiother Oncol 2013; 107: 325-332.

19. National Institutes of Health. Common Terminology Criteria for Adverse Events (CTCAE). Version 4.03.2010.

20. Chin J, Rumble RB, Kollmeier M et al. Brachytherapy for patients with prostate cancer: American Society of Clinical Oncology/Cancer Care Ontario Joint Guideline Update. J Clin Oncol 2017; 35: 1737-1743.

21. Sathya JR, Davis IR, Julian JA et al. Randomized trial comparing iridium implant plus external-beam radiation therapy with external-beam radiation therapy alone in node-negative locally advanced cancer of the prostate. J Clin Oncol 2005; 23: 1192-1199.

22. Dayes IS, Parpia S, Gilbert J et al. Long-term results of a randomized trial comparing iridium implant plus external beam radiation therapy with external beam radiation therapy alone in node-negative locally advanced cancer of the prostate. Int J Radiat Oncol Biol Phys 2017; 99: 90-93.

23. Hoskin PJ, Rojas AM, Bownes PJ et al. Randomised trial of external beam radiotherapy alone or combined with high- 
dose-rate brachytherapy boost for localised prostate cancer. Radiother Oncol 2012; 103: 217-222.

24. King MT, Yang DD, Muralidhar V et al. A comparative analysis of overall survival between high-dose-rate and lowdose-rate brachytherapy boosts for unfavorable-risk prostate cancer. Brachytherapy 2019; 18: 186-191.

25. Slevin F, Rodda SL, Bownes P et al. A comparison of outcomes for patients with intermediate and high risk prostate cancer treated with low dose rate and high dose rate brachytherapy in combination with external beam radiotherapy. Clin Transl Radiat Oncol 2020; 20: 1-8.

26. Morris WJ, Tyldesley S, Rodda S et al. Androgen suppression combined with elective nodal and dose escalated radiation therapy (the ASCENDE-RT Trial): an analysis of survival endpoints for a randomized trial comparing a low-dose-rate brachytherapy boost to a dose-escalated external beam boost for high- and intermediate-risk prostate cancer. Int J Radiat Oncol Biol Phys 2017; 98: 275-285.

27. Kee DLC, Gal J, Falk AT et al. Brachytherapy versus external beam radiotherapy boost for prostate cancer: Systematic review with meta-analysis of randomized trials. Cancer Treat Rev 2018; 70: 265-271.

28. Mendez L, Morton G. High dose-rate brachytherapy in the treatment of prostate cancer. Transl Androl Urol 2018; 7: 357370.

29. Yaxley J, Lah K, Yaxley J et al. Long-term outcomes of highdose-rate brachytherapy for intermediate- and high-risk prostate cancer with a median follow-up of 10 years. BJU Int 2017; 120: 56-60.

30. Joseph N, Taylor C, O'Hara C et al. A combined single highdose rate brachytherapy boost with hypofractionated external beam radiotherapy results in a high rate of biochemical disease free survival in localised intermediate and high risk prostate cancer patients. Radiother Oncol 2016; 121: 299-303.

31. Kaprealian T, Weinberg V, Speight JL et al. High-dose rate brachytherapy boost for prostate cancer: comparison of two different fractionation schemes. Int J Radiat Oncol Biol Phys 2012; 82: 222-227.

32. Agoston P, Major T, Fröhlich G et al. Moderate dose escalation with single-fraction high-dose-rate brachytherapy boost for clinically localized intermediate- and high-risk prostate cancer: 5-year outcome of the first 100 consecutively treated patients. Brachytherapy 2011; 10: 376-384.

33. Prada P, González H, Fernández J et al. Biochemical outcome after high-dose-rate intensity modulated brachytherapy with external beam radiotherapy: 12 years of experience. BJU Int 2012; 109: 1787-1793.

34. Moran B, Rice S, Chhabra A et al. Long-term biochemical outcomes using cesium-131 in prostate brachytherapy. Brachytherapy 2019; 18: 800-805.

35. Martell K, Mendez LC, Chung HT et al. Results of 15 Gy HDR-BT boost plus EBRT in intermediate-risk prostate cancer: Analysis of over 500 patients. Radiother Oncol 2019; 141: 149-155.

36. Chao M, Joon DL, Khoo V et al. Combined low dose rate brachytherapy and external beam radiation therapy for intermediate-risk prostate cancer. J Med Imaging Radiat Sci 2019; 50: 82-86.

37. Spratt D, Zumsteg Z, Ghadjar P et al. Comparison of high-dose (86.4 Gy) IMRT vs. combined brachytherapy plus IMRT for intermediate-risk prostate cancer. BJU Int 2014; 114: 360-367.

38. Okamoto K, Okuyama K, Kohno N et al. Clinical outcomes of low-dose-rate brachytherapy based radiotherapy for intermediate risk prostate cancer. J Contemp Brachytherapy 2020; 12: 6-11.

39. Rodda S, Tyldesley S, Morris WJ et al. ASCENDE-RT: an analysis of treatment-related morbidity for a randomized trial comparing a low-dose-rate brachytherapy boost with a dose-escalated external beam boost for high- and intermediate-risk prostate cancer. Int J Radiat Oncol Biol Phys 2017; 98: 286-295

40. Sakurai T, Takamatsu S, Shibata S et al. Toxicity and clinical outcomes of single-fraction high-dose-rate brachytherapy combined with external beam radiotherapy for high-/very high-risk prostate cancer: A dosimetric analysis of toxicity. Ipn I Radiol 2020; 38: 1197-1208.

41. Büchser D, Casquero F, Espinosa JM et al. Late toxicity after single dose HDR prostate brachytherapy and EBRT for localized prostate cancer: Clinical and dosimetric predictors in a prospective cohort study. Radiother Oncol 2019; 135: 13-18.

42. Kollmeier MA, McBride S, Varghese M et al. Low-dose-rate brachytherapy combined with ultrahypofractionated radiation therapy for clinically localized, intermediate-risk prostate cancer: results from a prospective trial. Int J Radiat Oncol Biol Phys 2020; 108: 905-913.

43. Goy B, Burchette R, Soper M et al. Ten-year treatment outcomes of radical prostatectomy vs. external beam radiation therapy vs. brachytherapy for 1503 patients with intermediate-risk prostate cancer. Urology 2020; 136: 180-189.

44. Hervás A, Pastor J, Gonzalez C et al. Outcomes and prognostic factors in intermediate-risk prostate cancer: multi-institutional analysis of the Spanish RECAP database. Clin Transl Oncol 2019; 21: 900-909.

45. Boladeras A, Martinez E, Ferrer F et al. Localized prostate cancer treated with external beam radiation therapy: longterm outcomes at a European comprehensive cancer centre. Rep Pract Oncol Radiother 2016; 21: 181-187.

46. Frank SJ, Pugh TJ, Blanchard P et al. Prospective phase 2 trial of permanent seed implantation prostate brachytherapy for intermediate-risk localized prostate cancer: efficacy, toxicity, and quality of life outcomes. Int J Radiat Oncol 2018; 100: 374-382.

47. Pons-Llanas O, Collado-Ballesteros E, Roldan-Ortega S et al. Impact of real-time, dose-escalated permanent seed implant brachytherapy in intermediate-risk prostate cancer. Rep Pract Oncol Radiother 2020; 25: 463-469.

48. Preisser F, Cooperberg MR, Crook J et al. Intermediate-risk prostate cancer: stratification and management. Eur Urol Oncol 2020; 3: 270-280.

49. Mendez LC, Martell K, Warner A et al. Does ADT benefit unfavourable intermediate risk prostate cancer patients treated with brachytherapy boost and external beam radiotherapy? A propensity-score matched analysis. Radiother Oncol 2020; 150: $195-200$.

50. Keyes M, Merrick G, Frank SJ et al. American Brachytherapy Society Task Group Report: Use of androgen deprivation therapy with prostate brachytherapy - a systematic literature review. Brachytherapy 2017; 16: 245-265.

51. Morton G, Loblaw A, Cheung P et al. Is single fraction 15 Gy the preferred high dose-rate brachytherapy boost dose for prostate cancer? Radiother Oncol 2011; 100: 463-467.

52. Merrick GS, Wallner KE, Galbreath RW et al. Is supplemental external beam radiation therapy essential to maximize brachytherapy outcomes in patients with unfavorable intermediate-risk disease? Brachytherapy 2016; 15: 79-84. 